\title{
Adaptive Coherent/Non-Coherent Spatial Modulation Aided Unmanned Aircraft Systems
}

\author{
Chao Xu, Member, IEEE, Tong Bai, Student Member, IEEE, Jiankang Zhang, Senior Member, IEEE, Rakshith \\ Rajashekar, Senior Member, IEEE, Robert G. Maunder, Senior Member, IEEE, Zhaocheng Wang ${ }^{1}$, Senior \\ Member, IEEE, and Lajos Hanzo* Fellow, IEEE
}

\begin{abstract}
Unmanned Aerial Vehicles (UAVs) are envisioned to be an important part of the device-centric Internet-of-Things (IoT). These bespoke Unmanned Aircraft Systems (UASs) that support UAVs significantly differ from traditional terrestrial and aeronautical networks, both of which are evolving towards their next-generation forms. The major challenges of the UAS include (1) the augmented interference due to strong Line-ofSight (LoS) (2) the dynamic shadowing effects owing to 3D aerial maneuvering, (3) the excessive Doppler shift owing to high UAV mobility as well as (4) the Size, Weight, And Power (SWAP) constraints. Against this background, we propose to invoke the recently developed coherent/non-coherent Spatial Modulation (SM) and its diversity-oriented counterpart of SpaceTime Block Coding using Index Shift Keying (STBC-ISK). These arrangements employ multiple Transmit Antennas (TAs) in order to improve the network's Quality-of-Service (QoS), but they only use a single RF chain. Furthermore, based on the throughput, delay and power-efficiency, we conceive a novel three-fold adaptivity design, where the UAS may adaptively (I) switch between coherent and non-coherent schemes, (II) switch between single- and multiple-TA based arrangements as well as (III) switch between high-diversity and high-spectral-efficiency multiple-TA based schemes.
\end{abstract}

\section{INTRODUCTION}

Following the success of the Wright brothers' manned flight in 1903, Unmanned Aerial Vehicles (UAVs) were first deployed in World War I for collecting intelligence and for attacks. The military motivations of advancing warfare advantage, reducing cost and saving lives of the pilots, who would otherwise be put at risk, have initiated the early development of UAVs. More than a century has passed by, and the rapidly proliferating UAVs are soon expected to swarm in the sky. However, the contemporary UAV applications are predominately motivated by civilian demands. This significant shift in the aircraft population and mission is regonized by the Federal Aviation Administration (FAA), who predicts that in 2030, the number of Remotely Operated Aircraft (ROA) or UAVs in the US may approach 20,000 [1]. In fact, over a million commercial UAVs had already been sold by 2015 ,

L. Hanzo would like to acknowledge the financial support of the Engineering and Physical Sciences Research Council projects EP/Noo4558/1, EP/PO34284/1, COALESCE, of the Royal Societys Global Challenges Research Fund Grant, of the Royal Society Grant IF170002 as well as of the European Research Councils Advanced Fellow Grant QuantCom.

C. Xu, J. Zhang, R. Rajashekar, R. G. Maunder and L. Hanzo are with the School of Electronics and Computer Science, University of Southampton, Southampton SO17 1BJ, UK (e-mail: \{cx1g08, jz09v, rmr1u14, rm, lh\}@soton.ac.uk). T. Bai is with the School of Electronic Engineering and Computer Science, Queen Mary University of London, London E1 4NS, UK (email: t.bai@qmul.ac.uk). Z. Wang is with Tsinghua University, Beijing, China (email: zcwang@tsinghua.edu.cn). which substantially outnumbered both manned aircraft and military UAVs.

The UAVs flying in both the controlled and uncontrolled airspace may conduct a variety of civilian missions, including but not limited to performing search-and-rescue, crowdmonitoring and environmental surveillance, transporting goods and information as well as providing seemless network coverage. Owing to their convenient deployability and diverse functions, the UAVs are expected to become an important part of the device-centric Internet-of-Things (IoT), where billions of smart devices will be connected in order to provide smart integrated services in support of the smart home, intelligent healthcare and smart transportation.

Driven by this escalating communication demand, the standardization of 5th Generation (5G) mobile networks is well underway. On the other hand, in order to improve the existing aeronautical networks, the modernization of Air Traffic Management (ATM) is undertaken by the Single European Sky ATM Research (SESAR) in Europe and by the Next Generation Air Transportation System (NextGen) in the US. The European and American aviation authorities [1] plan to complete the first upgrade phase in 2020 , which mainly aims for improving the existing communication links. Following this, the second phase will focus on data link services, where the principle of "Management by Intervention" in the first phase will be replaced by a more strategic "Management by Planning and Intervention by Exception" [1]. This implies that the future aeronautical networks would also follow the IoT philosophy, where the human-initiated management and intervention will be largely replaced by autonomous and smart systems.

The terminology of Unmanned Aircraft System (UAS) is adopted both by SESAR and by NextGen as the system supporting UAVs, regardless of UAV size. Both the $5 \mathrm{G}$ networks and the next-generation ATM are developing standards for integrating UAS. The UAS is generally constituted by a control link and a data link, which are termed as the Command \& Control (C\&C) link and the application data link by the 3rd Generation Partnership Project (3GPP) [2]. However, the UAS control link is termed as Control and Non-Payload Communication (CNPC) link in ATM [3]. The American ATM has granted both the L-band (960-977 MHz) and the C-band (5.03-5.091 GHz) to the CNPC link [3], whereas the European ATM has reserved the C-band (5.0$5.15 \mathrm{GHz}$ ) for UAS usage. In summary, although the Qualityof-Service (QoS) requirements for the data link may vary depending on the specific application, the ultra-reliability, ultra-robustness and ultra-low-latency of the control link is 
always of paramount importance.

Nonetheless, the UAS significantly differs from traditional terrestrial and aeronautical networks. Firstly, owing to the strong Line-of-Sight (LoS) propagation, the UAS is prone to inflicting increased interference upon the ground-level User Equipments (UEs) [2]. Secondly, owing to their dynamic aerial maneuvers especially in mission-critical applications, the critical air-ground link may become blocked by the chassis of the rotary-ring UAV or by the fuselage of the fixed-ring UAV, which is termed as the airframe shadowing effect in [3]. Thirdly, the UAS often encounters an excessive Doppler shift due to the high UAV velocity. Lastly, the Size, Weight, And Power (SWAP) constraints of UAVs hinder the deployment of advanced Multiple-Input Multiple-Output (MIMO) techniques for mitigating the detrimental fading effects.

Against this background, we propose to invoke the recently developed coherent/non-coherent Spatial Modulation (SM) [4] and Space-Time Block Coding using Index Shift Keying (STBC-ISK) [5]. In a nutshell, firstly, thanks to the employment of multiple Transmit Antennas (TAs), the SM and STBC-ISK schemes achieve a spectral-efficiency gain and a transmit-diversity gain, respectively, which improve the network's QoS. We will demonstrate that compared to their conventional single-TA aided counterparts, the multiple-TA aided coherent/non-coherent SM and STBC-ISK are capable of achieving the target QoS requirements at a reduced transmit power, which reduces the UAV's power consumption and the interference imposed on other users. Secondly, thanks to the diversity gain, the detrimental airframe shadowing effect is shown to be mitigated by the STBC-ISK arrangement. Thirdly, the noncoherent schemes of Differential SM (DSM) [6] and Differential STBC-ISK (DSTBC-ISK) [5] completely dispense with channel estimation, hence they are more robust to high Doppler frequencies. Lastly, unlike many conventional MIMO schemes such as the V-BLAST and STBC [7], the coherent/non-coherent schemes of SM and STBC-ISK always only activate a single RF chain, which reduces the deployment cost and energy dissipation compared to full-RF MIMO schemes.

Furthermore, all operational and future wireless communication systems are essentially adaptive. One of the most prominent strategies is the Adaptive Coding and Modulation (ACM), which adjusts both the modulation throughput and the channel coding rate according to the link quality. In order to better accommodate the high dynamics of the UAS, based on the throughput, delay and power-efficiency, we conceive a three-fold adaptivity design, where the UAS may adaptively (1) switch between coherent and non-coherent schemes at low and high normalized Doppler frequencies $f_{d}$, respectively, (2) reconfigure itself between single- and multiple-TA based arrangements at low and high channel coding rates $R_{c}$, respectively, and (3) switch between high-diversity and highspectral-efficiency multiple-TA based schemes at low and high modulation throughputs $R_{m}$, respectively.

\section{System OVERVIEW}

The physical attributes as well as the communication characteristics are summarized in Fig. 1, which shows that the
UAS strikes a fundamental tradeoff between terrestrial and aeronautical networks. The evolved versions of 5G and ATM, both of which aim for integrating UAS, open up compelling opportunities for the UAS and its IoT applications.

Although balloons are sometimes not considered as UAVs in the contemporary applications, they are actually the first ones that executed unmanned aerial missions - Austria used bomb-filled unmanned balloons to attack Venice in 1849. Today, there is an increasing interest in employing balloons or airships as static aerial BSs, which aim for providing network coverage in remote or disaster-stricken areas. Notably, Google's Project Loon uses high-altitude balloons operating in the stratospace above the altitude where airplanes fly, whereas the ABSOLUTE project in Europe employs low-altitude balloons operating in uncontrolled airspace [8]. Fig. 1 indicates that the elevated ballon/airship altitude results in favorable LoS conditions associated with reduced terrain shadowing and multipath fading. Moreover, the static aerial BS typically does not experience airframe-induced fading and high Doppler shift.

The FAA regulates small commercial UAVs to fly under $400 \mathrm{ft}$ of altitude and under $100 \mathrm{mph}$ of speed, which are characteristics of the popular rotary-wing quadcopter seen in Fig. 1, whereas 3GPP considers to support a higher altitude of $300 \mathrm{~m} \mathrm{[2].} \mathrm{As} \mathrm{an} \mathrm{aerial} \mathrm{UE,} \mathrm{the} \mathrm{UAV} \mathrm{is} \mathrm{prone} \mathrm{to} \mathrm{impose}$ increased interference to other ground-level UEs [2], owing to the strong LoS seen in Fig. 1. Moreover, Fig. 1 also shows that the near-ground UAS often experiences terrain shadowing and multipath fading [9]. The detrimental airframe shadowing in Fig. 1 arises, when the communication link is blocked by the UAV's chassis during its aerial maneuvers, resulting in significant packet loss [9]. Furthermore, Fig. 1 suggests that the control link of rotary-wing UAV may experience a high normalized Doppler frequency $f_{d}$. For example, the off-the-shelf XBee-PRO employed for the UAS control link in [10] operates at a carrier frequency of $f_{c}=2.4 \mathrm{GHz}$ and symbol rate of $f_{s}=9600 \mathrm{Bd}$, which exhibits a high $f_{d}=\frac{v \cdot f_{c}}{c \cdot f_{s}}=0.025$ for a UAV speed of $v=30 \mathrm{~m} / \mathrm{s}$, where $c=3 \times 10^{8} \mathrm{~m} / \mathrm{s}$ refers to the speed of light. The excessive $f_{d}$ may result in substantial Channel State Information (CSI) estimation overhead and CSI-error, which degrade the QoS of the control link upon invoking coherent communication techniques.

The fixed-wing UAVs seen in Fig. 1 exhibit the highest variations in altitude, speed and mission type. Firstly, their elevated altitudes result in an improved LoS, but the UAVs are still prone to experience terrain shadowing and multipath fading, because their near-ground operations do not share the same benign open-area airport environment as in conventional civil aviation. Secondly, the operational LTEadvanced systems are designed to offer services up to a highspeed train-velocity of $500 \mathrm{~km} / \mathrm{h}$, whereas the aeronautical networks supporting commercial flights generally support a velocity below the speed of sound of $1192 \mathrm{~km} / \mathrm{h}$. However, the record-holding hypersonic UAV may reach a whopping speed of 20 times higher than the speed of sound. Thirdly, without having to accommodate humans, the fixed-wing UAVs may perform more dramatic maneuvers for mission-critical applications, which induces a higher chance of encountering 


\begin{tabular}{|c|c|c|c|c|c|}
\hline & $\begin{array}{l}\text { Currently Upgrading } \\
5 G \text { Wireless Network }\end{array}$ & $<$ & Unmanned Aircraft Systems & 7 & $\begin{array}{l}\text { Currently Upgrading } \\
\text { Air Traffic Management }\end{array}$ \\
\hline & Terrestrial cellular & Balloon/Airship & Rotary-wing quadcopter & Fixed-wing glider/aircraft & Civil Aviation \\
\hline Altitude & Ground-level & $\begin{array}{c}\text { Low/High } \\
\text { (e.g. below } 1200 \mathrm{ft} / 365 \mathrm{~m} \\
\text { or above } 59000 \mathrm{ft} / 18 \mathrm{~km} \text { ) }\end{array}$ & $\begin{array}{c}\text { Low } \\
\text { (e.g. up to } 400 \mathrm{ft} / 120 \mathrm{~m} \\
\text { without licensing) }\end{array}$ & $\begin{array}{c}\text { Low } \rightarrow \text { High } \\
\text { (e.g. from near-ground } \\
\text { to stratospace) }\end{array}$ & $\begin{array}{c}\text { High } \\
\text { (e.g. up to } 59000 \mathrm{ft} / 18 \mathrm{~km} \\
\text { below stratospace) }\end{array}$ \\
\hline Speed & $\begin{array}{l}\text { Low } \\
\text { (e.g. up to } 310 \mathrm{mph} / 500 \mathrm{kmh} \\
\text { for high-speed train) }\end{array}$ & $\begin{array}{c}\text { Low } \\
\text { (near static) }\end{array}$ & $\begin{array}{c}\text { Low } \\
\text { (e.g. up to } 100 \mathrm{mph} / 160 \mathrm{kmh} \text { ) }\end{array}$ & $\begin{array}{c}\text { Low } \rightarrow \text { High } \\
(\text { e.g. may even exceed speed } \\
\text { of sound } 741 \mathrm{mph} / 1192 \mathrm{kmh} \text { ) }\end{array}$ & $\begin{array}{c}\text { High } \\
\text { (e.g. generally under speed } \\
\text { of sound } 741 \mathrm{mph} / 1192 \mathrm{kmh} \text { ) }\end{array}$ \\
\hline Dynamic Maneuver & Low & Low & High & High & High \\
\hline LOS Strength & Low & High & High & High & High \\
\hline Terrain Shadowing & High & Low & High for near-ground & High for near-ground & Low \\
\hline Multipath fading & High & Low & High for near-ground & High for near-ground & $\begin{array}{l}\text { High during taxiing, } \\
\text { taking-off, landing }\end{array}$ \\
\hline $\begin{array}{r}\text { Airframe Shadowing } \\
\text { (During Maneuver) }\end{array}$ & None & None & High for maneuver & High for maneuver & Low for gentle maneuver \\
\hline $\begin{array}{c}\text { Doppler Frequency } \\
\text { (Normalized by Symbol Rate) }\end{array}$ & Low & Low & High for control link & High for control link & High \\
\hline
\end{tabular}

Fig. 1: Communication systems from terrestrial cellular, Unmanned Aircraft Systems (UASs) to civil aviation.

the airframe shadowing effect. Lastly, for example, a generic CNPC testbed [3] recorded a very high $f_{d}=0.031$ for the 1023-chip m-sequence signals associated with a chip rate of $50 \mathrm{MHz}$ operating in the C-band at $f_{c}=5.06 \mathrm{GHz}$ and at a speed of $v=90 \mathrm{~m} / \mathrm{s}$.

In civil aviation, the detrimental effects of interference, aerial dynamics and mobility are generally mitigated by a high transmit power, so that a sufficiently high SNR is maintained throughout all phases of a flight. By contrast, the UAVs often have to use lightweight lithium-ion batteries, which are responsible for powering the whole platform. The pertinent SWAP constraints hinder the employment of both high-gain steerable but bulky antennas and of full-RF MIMO schemes. Against this background, in this work, we characterize the air-ground channels for UAS by the stochastic Ricean fading model, where the suitable single-RF techniques are adaptively selected based on the Doppler frequency, Ricean $K$-factor and SNR. It is worth noting that the free-space and two-ray path loss models are generally determinstic and very slow-changing. For example, even with a high UAV speed of $200 \mathrm{~m} / \mathrm{s}$ and a typical bit rate of $100 \mathrm{kbps}$, the distance change is merely $0.002 \mathrm{~m}$ over a bit duration, which is negligible compared to thousands of meters of LoS propagation coverage. Therefore, the path loss models are helpful for link budget design but often indifferent to the choice of PHY-layer techniques for UAS.

\section{Single-RF Design: The Minimum Hardware EXPENDITURE}

The schematics of the Index Modulation (IM) schemes of SM [4] and STBC-ISK [5] are portrayed in Figs. 2(a) and 2(b), respectively. More explicitly, based on the V-BLAST signal structure of $\mathbf{s}=\left[s_{1}, s_{2}\right]$, the SM of Fig. 2(a) assigns one IM bit to decide whether to transmit $\mathbf{s}=\left[s^{l}, 0\right]$ or $\mathbf{s}=\left[0, s^{l}\right]$, where the $L$ PSK symbol $s^{l}$ is modulated by $\log _{2} L$ bits. As a result, the coherent SM scheme achieves an improved spectralefficiency of $R=\log _{2} L+1$, which is higher than the singleTA aided PSK of $R=\log _{2} L$, as shown in Fig. 2(c). Similarly, based on the Alamouti's G2 structure of $\mathbf{S}=\left[\begin{array}{cc}s_{1} & s_{2} \\ -s_{2}^{*} & s_{1}^{*}\end{array}\right]$, the STBC-ISK of Fig. 2(b) assigns one IM bit to decide whether to transmit $\mathbf{S}=\left[\begin{array}{cc}s^{l} & 0 \\ 0 & \left(s^{l}\right)^{*}\end{array}\right]$ or $\mathbf{S}=\left[\begin{array}{cc}0 & s^{l} \\ -\left(s^{l}\right)^{*} & 0\end{array}\right]$. The spectralefficiency of STBC-ISK is given by $R=\frac{\log _{2} L+1}{2}$, which is lower than the single-TA scheme, but the STBC-ISK achieves a beneficial diversity gain, as seen in Fig. 2(c).

The non-coherent single-TA-based DPSK seen in Fig. 2(c) invokes the differential encoding of $s_{n}=x_{n-1} s_{n-1}$, where $x_{n-1}$ is the modulated LPSK symbol. The matrix-based differential encoding for the multiple-TA schemes is given by $\mathbf{S}_{n}=\mathbf{X}_{n-1} \mathbf{S}_{n-1}$, where the $(T \times M)$-element matrix $\mathbf{S}_{n}$ models the signals transmitted by $M$ TAs over $T$ time slots. In order to form a $(T \times T)$-element unitary matrix $\mathbf{X}_{n-1}$, the DSM scheme [6] invokes $\mathbf{X}_{n-1}=\left\{\left[\begin{array}{cc}x_{1} & 0 \\ 0 & x_{2}\end{array}\right],\left[\begin{array}{cc}0 & x_{1} \\ x_{2} & 0\end{array}\right]\right\}$ for $M=T=2$, where $s_{1}$ and $s_{2}$ are two independently modulated LPSK symbols, while a single IM bit is assigned to determine the activation order. On the other hand, the DSTBC-ISK scheme uses the IM aided STBC matrices of $\mathbf{X}_{n-1}=\left\{\begin{array}{cc}x^{l} & 0 \\ 0 & \left(x^{l}\right)^{*}\end{array}\right],\left[\begin{array}{cc}0 & x^{l} \\ -\left(x^{l}\right)^{*} & 0\end{array}\right\}$. For $M=2$, the spectralefficiencies of DSM and DSTBC-ISK are $R=\log _{2} L+\frac{1}{2}$ and $R=\frac{\log _{2} L+1}{2}$, respectively, as shown in Fig. 2(c). Interested readers may refer to [4]-[7] for the extensions of using $M>2$.

The single-RF design reduces the cost for the following reasons. Firstly, instead of employing the full-RF V-BLAST and STBC, the SM and STBC-ISK arrangements also achieve a spectral-efficiency gain and a diversity gain, respectively, but they only use a single RF chain, as highlighted in Fig. 2(c). Secondly, the inter-antenna synchronization is eliminated. Thirdly, in the absence of Inter-Antenna Interference (IAI), the coherent/non-coherent SM and STBC-ISK schemes may 


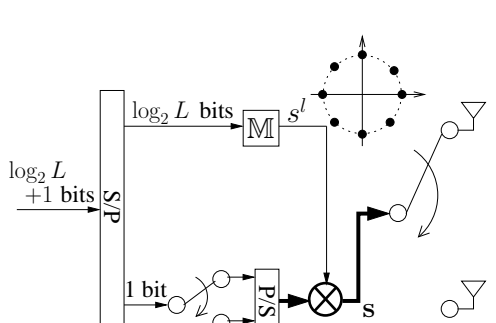

(a) Coherent SM

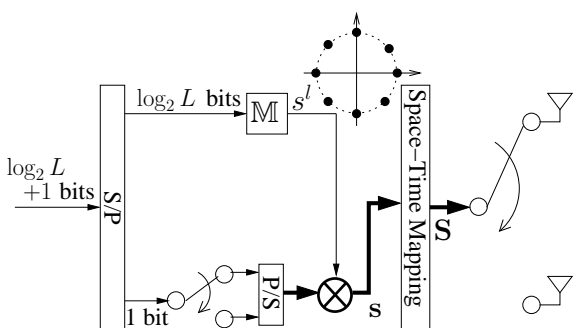

(b) Coherent STBC-ISK

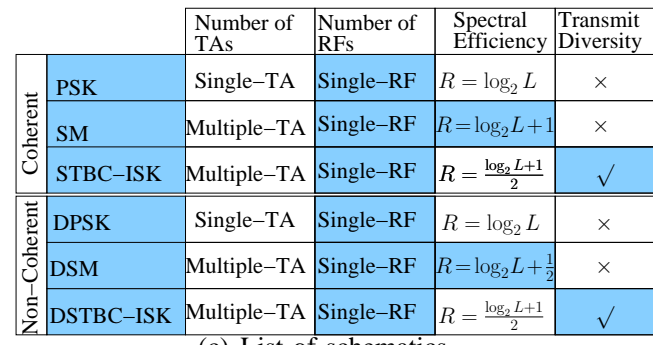

(c) List of schematics

Fig. 2: Schematics of Spatial Modulation (SM) [4] and Space-Time Block Code using Index Shift Keying (STBC-ISK) [5] as well as the list of schemes considered in this paper.

employ single-stream-based signal detection at the receiver [7], which reduces both the signal processing complexity and latency.

Moreover, we note that the non-coherent MIMO schemes often suffer from the so-called infinite-cardinality problem discussed in [5], where the transmit signals have an infinite number of arbitrary phases and magnitudes. This imposes extra constraints on the Digital-to-Analog Converter (DAC), which is appropriately matched to the finite number of constellation points to generate the corresponding analog signals. Furthermore, the linear amplication of the associated signals having a high Peak-to-Average Power Ratio (PAPR) requires a substantial Power Amplifer (PA) Input Back-Off (IBO) [11]. On one hand, reducing the IBO imposes in-band signal distortion that results in performance degradation. On the other hand, increasing the IBO leads to low PA efficiency and out-of-band power leakage contaminating the adjacent channels. In order to extend the battery life and to avoid imposing interference on the other users, we opt for using constant-envelop PSK having a beneficial PAPR for all the UAS schemes summarized in Fig. 2(c).

\section{Airframe Shadowing}

The three-axis maneuver control including pitch, roll and yaw portrayed in Figs. 3(a) and 3(b) was key to the success of the Wright brothers flight in 1903. However, the dynamic maneuvers may induce detrimental airframe shadowing, which is observed to last as long as 74 seconds for the fixed-wing UAV [3]. This is highly hazardous as the UAV may have travelled thousands of meters with a blocked control link. Furthermore, it is also reported in [3] that using multiple antennas at the Ground Station (GS) is unable to mitigate airframe shadowing. The traditional solution is to employ a pair of aircraft TAs radiating the same signal, which however would result in self-interference nulls. Against this background, Alamouti's G2 STBC scheme is invoked in [12], where the signals transmitted from two TAs over two symbol periods are combined constructively thanks to the STBC's beneficial transmit diversity design.

When airframe shadowing is encountered, the pair of Ricean $K$-factors $K_{1}$ and $K_{2}$ experienced by the two TAs are subjected to non-negative log-normal distribution as modelled in [3]. Moreover, Fig. 3(a) shows that terrain shadowing may also result in independent LoS signal-strengths of $K_{1} \neq K_{2}$ for low-altitude UAVs, where the maneuver pattern of throttle has a substantial impact. In summary, we recommend the UAS

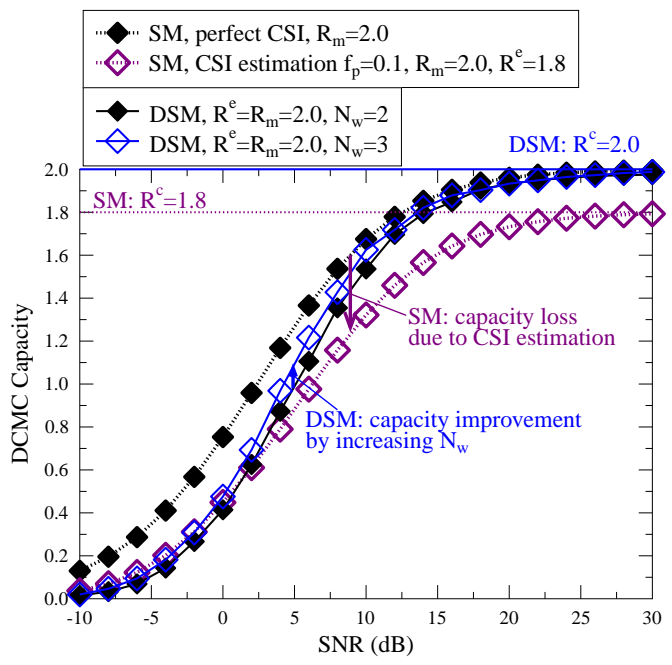

(a) Coherent/Differential SM

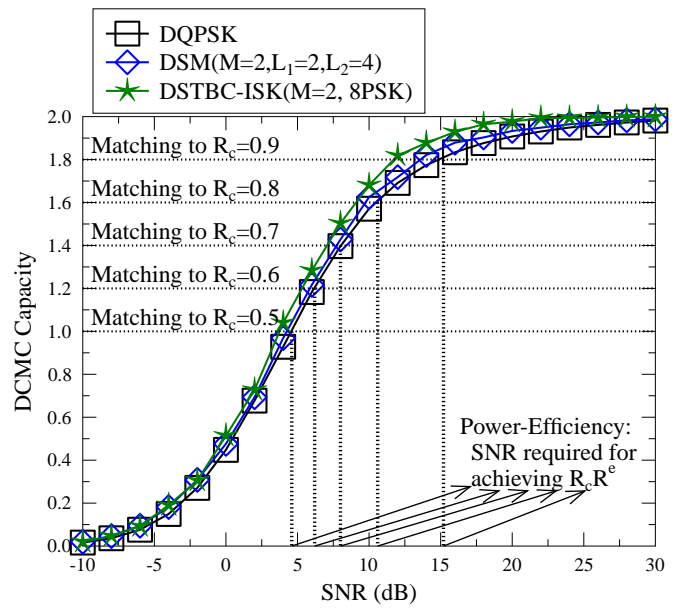

(b) Single-/Multiple-TAs, $N_{w}=3$

Fig. 4: DCMC capacity comparison of coherent/non-coherent SM as well as sinlge-/multiple-TA schemes over Ricean fading channels $\left(K=0 \mathrm{~dB}, f_{d}=0.03\right)$.

to employ the newly-developed single-RF scheme of STBCISK, which is shown in Fig. 3(c) to perform very closely to the full-RF STBC. Furthermore, as evidenced in Fig. 3(c) at the BER level of $10^{-4}$, the STBC-ISK is capable of achieving a substantial $13 \mathrm{~dB}$ performance advantage over its single-TAbased counterpart.

\section{Quality-OF-SERVICE (QoS): ThroughPUT}

Let us now proceed to examine the QoS of UAS. The modulation throughput and channel coding rate are denoted by $R_{m}$ 


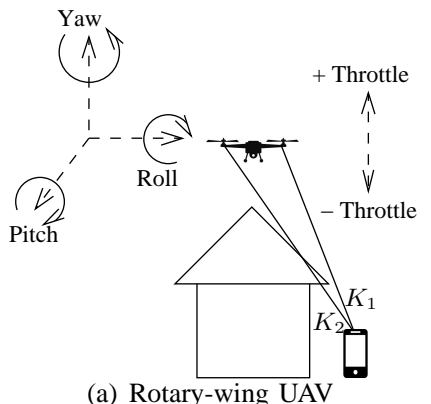

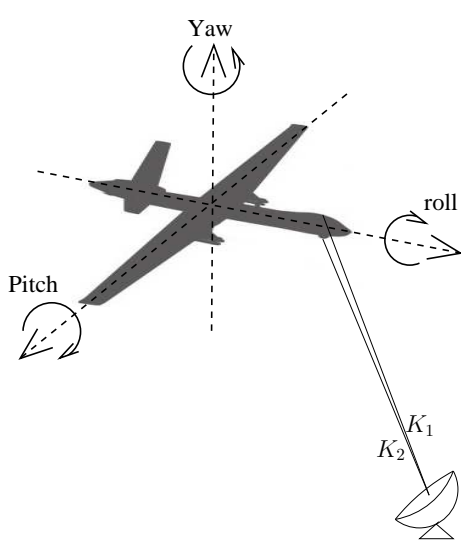

(b) Fixed-wing UAV

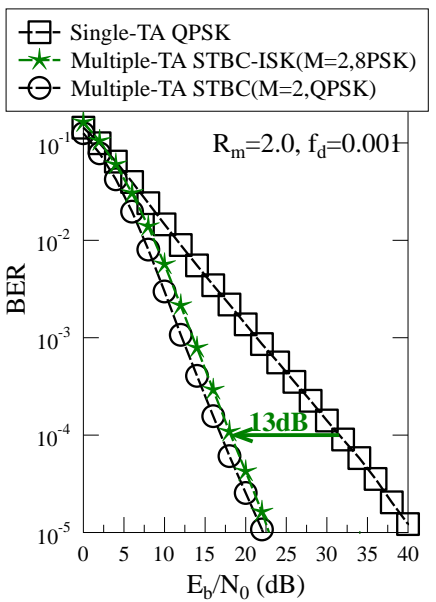

(c) BER performance

Fig. 3: Illustration of UAV maneuver and BER performance of single-/multiple-TA based schemes over Ricean fading channels associated with $\left\{K_{1} \neq K_{2}\right\} \in 10-|C N(0,10)| \mathrm{dB}$ and $f_{d}=0.001$.

and $R_{c}$, respectively. The effective throughputs of coherent and non-coherent schemes are given by $R^{e}=\left(1-f_{p}\right) R_{m}$ and $R^{e}=R_{m}$, respectively, where the pilot percentage of $f_{p}=0.1$ is used for channel estimation. The Ricean $K$-factor is generally assumed to be $K=0 \mathrm{~dB}$, because the value of $K$-factor does not affect the adaptivity decisions, which will be demonstrated in Sec. VII.

Moreover, we invoke the state-of-the-art Multiple-Symbol Differential Detector (MSDD) of [13] for non-coherent signal detection. Considering DPSK as an example, which invokes the differential encoding of $s_{n}=x_{n-1} s_{n-1}$, the simple Conventional Differential Detection (CDD) may recover $\hat{x}_{n-1}$ based on the phase difference between the consecutive received samples $\angle\left(y_{n} / y_{n-1}\right)$. However, the CDD suffers from an error floor at high Doppler frequency. As a remedy, MSDD associated with an increased window size of say $N_{w}=3$ makes a joint decision on $\left\{\hat{x}_{n-1}, \hat{x}_{n-2}\right\}$ based on $\left(N_{w}=3\right)$ observations $\left\{y_{n}, y_{n-1}, y_{n-2}\right\}$. The MSDD window size $N_{w}$ may be further increased for a better performance, but the associated detection complexity also grows exponentially with $N_{w}$. Fortunately, the MSDD complexity may be mitigated either by a sphere/trellis decoder or by the classic decisionfeedback methodology.

The throughput is characterized by the Discrete-Input Continuous-Output (DCMC) capacity in Fig. 4. According to the Shannon-Hartley law, the channel capacity is given by $R=B \cdot I(X ; Y)$, where $B$ denotes the bandwidth, while the mutual information $I(X ; Y)$ is maximized for Gaussiandistributed continuous-input variable $X$ and continuous-output $Y$. However, when we consider the practical digital modulation schemes, the associated DCMC capacity $I(\bar{X} ; Y)$ portrayed in Fig. 4 is bounded by the effective throughput of $R^{e}$. More explicitly, Fig. 4(a) demonstrates that at a high $f_{d}=0.03$, the coherent SM suffers from a capacity loss both due to the $10 \%$ pilot-overhead cost and owing to the CSI estimation error. By contrast, the noncoherent DSM dispensing with channel estimation benefits from a further capacity improvement by MSDD. In summary, the coherent scheme requires a higher bandwidth than the differential scheme for the sake of achieving the same target data rate.

Furthermore, it is evidenced by Fig. 4(b) that the high-

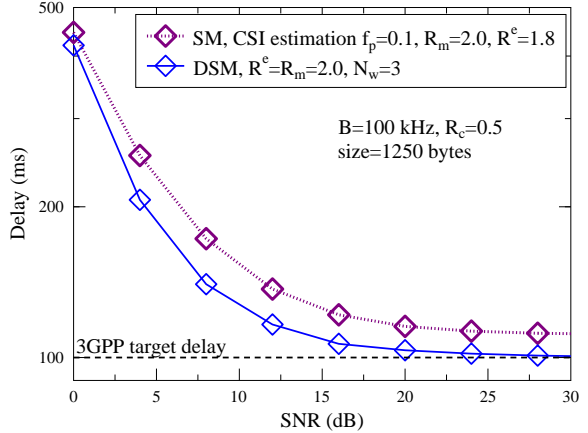

(a) Coherent/Differential SM

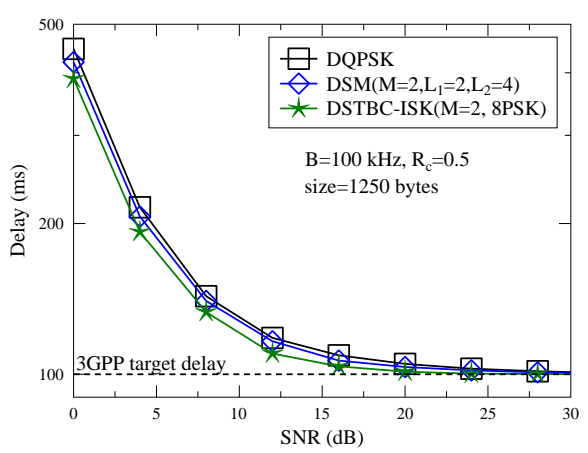

(b) Single-/Multiple-TAs, $N_{w}=3$

Fig. 5: Delay comparison of coherent/non-coherent SM as well as sinlge-/multiple-TA schemes over Ricean fading channels $\left(K=0 \mathrm{~dB}, f_{d}=0.03\right)$.

diversity scheme of DSTBC-ISK achieves the best DCMC capacity, followed by the high-spectral-efficiency DSM and the single-TA-based DQPSK. Moreover, we note that in channel coded scenarios, the power-efficiency is quantified by the SNR required for achieving the maximum attainable rate of $R^{e} R_{c}$, which is exemplified for DQPSK in Fig. 4(b).

\section{Quality-OF-Service (QoS): Delay}

The 3GPP recommands a packet size of $b=1250$ bytes and a target delay of $100 \mathrm{~ms}$ for the UAS C\&C link [2]. We henceforth assume a clean single-user link bandwidth of $B=100 \mathrm{kHz}$, which is supposed to satisfy the 3GPP C\&C data rate of $100 \mathrm{kbps}$, when a modulation scheme of $R_{m}=2.0$ 


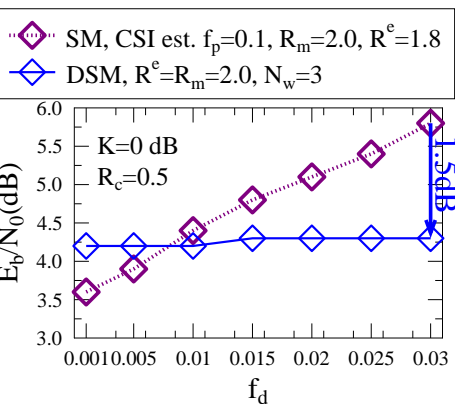

(a) Effect of $f_{d}$

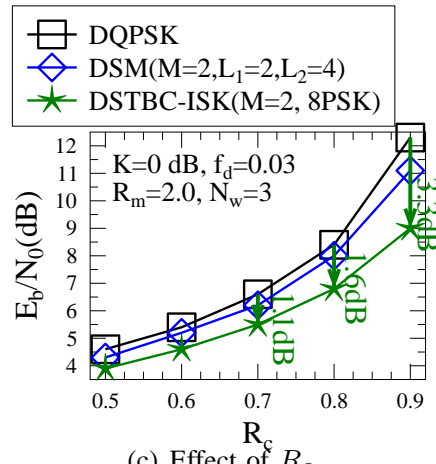

(c) Effect of $R_{c}$

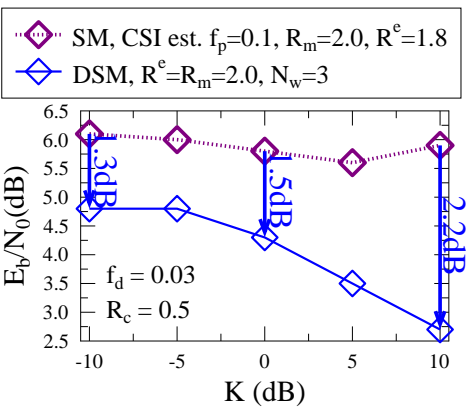

(b) Effect of $K$

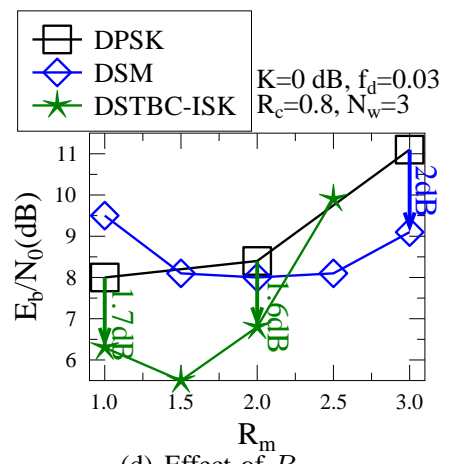

(d) Effect of $R_{m}$
Fig. 6: Comparison of the values of $E_{b} / N_{0}$ required for the coherent/non-coherent SM as well as sinlge-/multiple-TA schemes to achieve the maximum attainable rate of $R^{e} R_{c}$.

is used associated with a channel coding rate $R_{c}=0.5$. We note that this assumption is also sufficient for accommodating the ATM requirements [1], where the CNPC link data rate is estimated to be about $10 \mathrm{kbps}$.

As a result, the delay of Fig. 5 is evaluated by $\tau=$ $b /\left[B \cdot R_{c} \cdot I(\bar{X} ; Y)\right]$. More explicitly, Fig. 5(a) demonstrates that the 3GPP target delay is achieved by the noncoherent DSM, but it is not reached by the coherent SM due to its pilotoverhead cost and CSI estimation error. Moreover, Fig. 5(b) confirms that the DSTBC-ISK scheme performs the best, since it achieves the target delay at the lowest SNR, followed by DSM and DQPSK.

\section{QuAlity-OF-SERVICE (QoS): POWER-EFFICIENCY}

The QoS metric of power-efficiency is quantified in Fig. 6 by the $E_{b} / N_{0}$ required for achieving the maximum attainable rate of $R^{e} R_{c}$, which was exemplied earlier by Fig. 4(b). It is evidenced by Fig. 6(a) that the coherent SM performs better at low $f_{d}$, but its performance degrades substantially upon increasing $f_{d}$. By contrast, the noncoherent DSM scheme is shown in Fig. 6(a) to be robust to the increasing Doppler frequency. Therefore, we propose for the UAS to adaptively switch between coherent and non-coherent schemes. The switching threshold is suggested to be about $f_{d}=0.007$ according to Fig. 6(a), where a substantial power-efficiency gain of $1.5 \mathrm{~dB}$ is achieved at $f_{d}=0.03$. Moreover, Fig. 6(b) confirms that the different values of the Ricean $K$-factor do not affect the advantage of DSM over SM at $f_{d}=0.03$, where the power-efficiency gain is further enlarged upon increasing $K$.

The effect of $R_{c}$ is investigated in Fig. 6(c), which demonstrates that DSTBC-ISK and DSM perform similarly to DPSK, when a strong channel code of $R_{c}=0.5$ is applied. Nonetheless, it is also evidenced by Fig. 6(c) that DSTBC-ISK and DSM become more advantageous as $R_{c}$ increases, where the power-efficiency gain is as substantial as $3.3 \mathrm{~dB}$ at $R_{c}=0.9$. Therefore, we propose for the UAS to adaptively switch between single- and multiple-TA schemes, where the multipleTA scheme is used when a weaker channel code of $R_{c}>0.5$ is applied.

Lastly, as for the effect of $R_{m}$, Fig. 6(d) demonstrates that the DSTBC-ISK achieves power-efficiency gains of $1.7 \mathrm{~dB}$ and $1.6 \mathrm{~dB}$ over DPSK at $R_{m}=1.0$ and $R_{m}=2.0$, respectively. However, the performance of DSTBC-ISK degrades substantially for $R_{m}>2.0$. Nonetheless, Fig. 6(d) shows that the DSM is capable of achieving a substantial $2.0 \mathrm{~dB}$ powerefficiency gain over DPSK at $R_{m}=3.0$. Therefore, we further propose for the UAS to adaptively switch between the highdiversity and high-spectral-efficiency multiple-TA schemes, where DSTBC-ISK and DSM may be invoked for $R_{m} \leq 2.0$ and $R_{m}>2.0$, respectively.

\section{PRactical Considerations on Adaptivity}

The single-TA based ACM is adopted in the L-DACS2 link of the next-generation ATM. Moreover, the Rate Adaptation (RA) module that switches between diversity-oriented and multiplexing-oriented full-RF multiple-TA schemes has also been developed for 802.11 [14]. However, the full-RF multiple-TA schemes may be deemed less suitable for the UAS as discussed in Sec. II. By contrast, apart from having multiple TAs, the only extra cost of the SM and STBCISK arrangements in Fig. 2 is a RF switch that activates the TAs. The solution relies on the classic switch-mode PA design. For example, the auxiliary class-C PA of the Doherty architecture can be turned on and off at the symbol rate [11]. Therefore, these high-speed RF switching transistors can also be employed by the single-RF schemes of Fig. 2 for turning on and off the activated TA.

The adaptive switching decisions are generally made within the MAC-layer. The parameters of UAV speed, carrier frequency and symbol rate are all known by the UAV, hence the coherent/noncoherent adaptivity does not impose extra overhead by evaluating the Doppler frequency. Moreover, the single-/multiple-TA adaptivity and the diversity/spectralefficiency adaptivity are indexed by the SNR, as seen in Figs. 6(c) and (d), which can be directly incorporated into the existing RA algorithms [15]. We note that the popular RA scheme of SampleRate seen in [15] updates its adaptivity decisions in every 10 seconds, during which 100 packets are conveyed based on the C\&C example of Sec. VI. Considering the UAV's dynamic mobility, the adaptive probing interval of [14] that increases in proportion to the packet loss may be more suitable for the UAS applications.

\section{Conclusions}

We demonstrate that the recently developed coherent/noncoherent SM and its diversity-aided counterpart of STBC-ISK are capable of offering significant QoS improvements for UAS. Furthermore, we devised a novel three-fold 
adaptivity design, where the UAS may adaptively (1) switch between coherent and non-coherent schemes based on the Doppler frequency, (2) reconfigure itself between single- and multiple-TA schemes based on the channel coding rate as well as (3) switch between high-diversity and high-spectralefficiency multiple-TA schemes based on the modulation throughput.

\section{REFERENCES}

[1] EUROCONTROL/FAA Future Communications Study Operational Concepts and Requirements Team, "Communications operating concept and requirements (COCR) for the future radio system," Tech. Rep. 1.0.

[2] 3GPP Technical Report 36.777, "Technical specification group radio access network; study on enhanced LTE support for aerial vehicles (release 15)," [Online]. Available: http://www.3gpp.org/ftp//Specs/archive/36_series/36.777/, Jan. 2018.

[3] R. Sun, D. W. Matolak, and W. Rayess, "Air-ground channel characterization for unmanned aircraft systems - part IV: Airframe shadowing," IEEE Trans. Veh. Technol., vol. 66, pp. 7643-7652, Sept 2017.

[4] R. Y. Mesleh, H. Haas, S. Sinanovic, C. W. Ahn, and S. Yun, "Spatial modulation," IEEE Trans. Veh. Technol., vol. 57, pp. 2228-2241, July 2008.

[5] C. Xu, R. Rajashekar, N. Ishikawa, S. Sugiura, and L. Hanzo, "SingleRF index shift keying aided differential space-time block coding," IEEE Trans. Signal Process., vol. 66, pp. 773-788, Feb 2018.

[6] Y. Bian, X. Cheng, M. Wen, L. Yang, H. V. Poor, and B. Jiao, "Differential spatial modulation," IEEE Trans. Veh. Technol., vol. 64, pp. 3262-3268, July 2015.

[7] C. Xu, S. Sugiura, S. X. Ng, P. Zhang, L. Wang, and L. Hanzo, "Two decades of MIMO design tradeoffs and reduced-complexity MIMO detection in near-capacity systems," IEEE Access, vol. 5, pp. 18564 $18632,2017$.

[8] S. Chandrasekharan, K. Gomez, A. Al-Hourani, S. Kandeepan, T. Rasheed, L. Goratti, L. Reynaud, D. Grace, I. Bucaille, T. Wirth, and S. Allsopp, "Designing and implementing future aerial communication networks," IEEE Commun. Mag., vol. 54, pp. 26-34, May 2016.

[9] S. Hayat, E. Yanmaz, and R. Muzaffar, "Survey on unmanned aerial vehicle networks for civil applications: A communications viewpoint," IEEE Commun. Surveys Tuts., vol. 18, pp. 2624-2661, Fourthquarter 2016.

[10] T. Andre, K. A. Hummel, A. P. Schoellig, E. Yanmaz, M. Asadpour, C. Bettstetter, P. Grippa, H. Hellwagner, S. Sand, and S. Zhang, "Application-driven design of aerial communication networks," IEEE Commun. Mag., vol. 52, pp. 129-137, May 2014.

[11] J. Joung, C. K. Ho, K. Adachi, and S. Sun, "A survey on poweramplifier-centric techniques for spectrum- and energy-efficient wireless communications," IEEE Commun. Surveys Tus, vol. 17, pp. 315-333, Firstquarter 2015.

[12] M. A. Jensen, M. D. Rice, and A. L. Anderson, "Aeronautical telemetry using multiple-antenna transmitters," IEEE Trans. Aerosp. Electron. Syst., vol. 43, pp. 262-272, January 2007.

[13] D. Divsalar and M. K. Simon, "Maximum-likelihood differential detection of uncoded and trellis coded amplitude phase modulation over awgn and fading channels-metrics and performance," IEEE Trans. Commun., vol. 42, pp. 76-89, Jan 1994.

[14] I. Pefkianakis, S. Lee, and S. Lu, "Towards MIMO-aware 802.11n rate adaptation," IEEE/ACM Trans. Networking, vol. 21, pp. 692-705, June 2013.

[15] S. Biaz and S. Wu, "Rate adaptation algorithms for IEEE 802.11 networks: A survey and comparison," in 2008 IEEE Symp. Comput. Commun., pp. 130-136, July 2008

Chao Xu (M'14) received a B.Eng. from Beijing University of Posts and Telecommunications, China, and a BSc(Eng) with First Class Honours from Queen Mary, University of London, UK, through a Sino-UK program in 2008. He obtained a MSc with distinction and a Ph.D. from the University of Southampton, UK in 2009 and 2015, respectively. He is currently a research fellow at University of Southampton.
Tong Bai (S15) received the B.Sc. in telecommunications from Northwestern Polytechnical University, Xian, China, in 2013, the M.Sc. and Ph.D. in wireline and wireless communications from University of Southampton, U.K. in 2014 and 2019, respectively. His research interests include performance analysis, transceiver design and utility optimization both for wireline and for wireless communications.

Jiankang Zhang (SM18) received the Ph.D. degree in Communication and Information Systems from Zhengzhou University in 2012. Dr. Zhang has been a lecturer from 2012 and an associate professor from 2013 in School of Information Engineering, Zhengzhou University. Since 2014, he has been a senior research fellow in the University of Southampton, UK. His research interests are in the areas of wireless communications and signal processing, aeronautical communications and broadband communications.

Rakshith Rajashekar (SM'17) received the B.E. degree in electrical communication engineering from Visvesvaraya Technological University, Karnataka, India, in 2007. He received his Ph.D. from the Department of Electrical Communication Engineering, Indian Institute of Science (IISc), India, in 2014. He is presently working as a Research Fellow at the University of Southampton, UK. His research interests include antenna selection in MIMO systems, differential communication, millimeter wave communication, communication between drones with a focus on space-time signal processing and coding.

Robert G. Maunder (SM12) received the B.Eng. degree (Hons.) in electronic engineering and the Ph.D. degree in telecommunications, from the School of Electronics and Computer Science, University of Southampton, U.K., in 2003 and 2007, respectively. He began a Lectureship, in 2007 and was promoted to an Associate Professor, in 2013 and to a Professor, in 2017, in the NGW Group in the School of Electronics and Computer Science, University of Southampton, U.K.. He is the Founder and CTO of AccelerComm Ltd., which is commercializing his research as soft-IP. His research interests include joint source/channel coding and the holistic design of algorithms and hardware implementations for wireless communications. He has published over 100 IEEE papers in these areas. He received a Chartered Engineer of the IET, in 2013, and a Fellow of the IET, in 2017.

Zhaocheng Wang has been a Professor with Dept. of Electronic Engineering, Tsinghua University since April 2009, where he is currently the Director of Broadband Communication Key Laboratory, Beijing National Research Center for Information Science and Technology (BNRist). He hold 34 US/EU granted patents, published over 150 peer-reviewed journal papers and co-authored two Wiley books. Prof. Wang received IEEE Scott Helt Memorial Award, IET Premium Award, IEEE ComSoc Asia-Pacific Outstanding Paper Award and ICC2013/ICC2017 Best Paper Award. He was an Associate Editor of IEEE Trans. Wireless Communications from 
2011 to 2015 and an Associate Editor of IEEE Communications Letters from 2013 to 2016.

Lajos Hanzo (F'04) FREng, FIEEE, FIET, Fellow of EURASIP, DSc, received his degree in electronics in 1976 and his doctorate in 1983. He holds an honorary doctorate from the Technical University of Budapest (2009) and from the University of Edinburgh (2015). He is a member of the Hungarian Academy of Sciences and a former Editor-in-Chief of the IEEE Press. He is a Governor of both IEEE ComSoc and of VTS. 